\title{
THE FUTURE OF TECHNOLOGY AND ROBOTICS IN SURGERY
}

\author{
Ismael Domínguez-Rosado and Miguel A. Mercado* \\ Departament of Surgery, Instituto Nacional de Ciencias Médicas y Nutrición Salvador Zubirán, Mexico City, Mexico
}

\begin{abstract}
In the past three decades, several technologies designed for other purposes, have been applied in surgery to provide more precision to the surgical procedures and better outcomes. In surgery, innovation requires evidence before widespread implementation of novelties and a continuous quality improvement process to assess benefits and risks. Robotics in surgery has been widely implemented, but in some cases, there are many doubts regarding its clinical benefit and cost utility. The future of surgery lies in the fulfillment of four main conditions: safety, access, efficiency, and efficacy. Innovation and technology should help to accomplish these conditions, but it must not be the center of surgical practice. We present here our perspective on the main issues related to technology and robotics focusing on evidence-based surgery. (REV INVEST CLIN. 2021;73(5):326-8)
\end{abstract}

Key words: Robotics. Surgery. New technologies in surgery.

As defined by Dr. Gawande ${ }^{1}$, surgery is a profession defined by its authority to cure by means of bodily invasion. For many years, the great problems of surgery were perioperative management (anesthetic and pain management) and infections. Many developments have been achieved so far in the past two centuries, making these issues reasonably straightforward to manage.

In the past three decades, several technologies designed for other purposes, have been applied to surgery with the aim of providing more precision and better surgical outcomes. Theoretically, these technologies have had a great impact on patient care. The Da Vinci robotic platform from Intuitive Inc. ${ }^{\otimes}$ has been approved by the Food and Drug Administration of the USA to assist on most abdominal surgical procedures.
Its main areas of use are surgical procedures that involve narrow and deep anatomic spaces in the human body, such as pelvic organs, liver, mediastinum, thoracic cavity, and head-and-neck areas that might be difficult to access from an open approach without morbid procedures. In addition, the robot allows the surgeon to operate in ergonomic posture, with superb 3-D view and no hand shaking at all. Despite the enormous room for improvement and applicability of robotic platforms and telesurgery, marketing has gone faster than evidence to support their use on many surgical procedures. As shown by Sheetz et al. ${ }^{2}$, robotic colectomy has increased more than $30 \%$ in the past decade, despite the evidence showing marginal benefit compared to laparoscopic approach and certainly higher costs. Recently, the robotic versus laparoscopic resection for rectal cancer trial ${ }^{3}$ failed to
*Corresponding author:

Miguel A. Mercado

E-mail: mercadiazma@yahoo.com
Received for publication: 02-06-2021

Approved for publication: 15-06-2021

DOI: $10.24875 / R I C .21000304$

0034-8376 / (C) 2021 Revista de Investigación Clínica. Published by Permanyer. This is an open access article under the CC BY-NC-ND license (http://creativecommons.org/licenses/by-nc-nd/4.0/). 
show advantages over laparoscopy in rates of conversion, intraoperative complications, mortality, and quality of life.

Starting a robotic surgical program requires carefully planned steps to assure safety for the patient. A learning curve is implied in the use of the platform; however, safety compromise is not justified for the sake of learning. Pancreas surgery has shown the importance of a well-designed training program with qualified mentors and proctors to achieve good surgical outcomes while minimizing the risk of complications related to the learning process. The contrary is evident when the robotic platform is applied without proper initial guidance and training. In fact, the early experience on robotic cholecystectomy in the state of New York, USA, showed an increase in conversions versus laparoscopic cholecystectomy ( $4.9 \%$ vs. $2.8 \%$ ), bile duct injury ( $1.3 \%$ vs. $0.4 \%$ ), major reconstructive interventions $(0.6 \%$ vs. $0.1 \%)$, readmission $(7.3 \%$ vs. $4.4 \%)$, and 12 -month surgery related costs ${ }^{4}$.

Embracing novelty is frequent among surgeons. Unfortunately, the drive from the industry to impose technology looking for an indication is strong. It is easy to get entangled with industry health practitioners' relationships full of conflicts of interest (COI), and robotic surgery is not the exception. A recent analysis of the accuracy of self-declared $\mathrm{COI}$ statements in robotic studies showed that from 458 publications, $52 \%$ had one or more authors which received undeclared payments from the robotic industry (Intuitive Inc.). Those studies with undeclared payments were more likely to recommend robotic surgery compared to those that declared $\mathrm{COIs}^{5}$. This shows that even when the relationship between the industry and health practitioners is important to advance medicine, more work is needed for the sake of transparency. Innovative is not equal to better. New devices and surgical strategies need to pass the test of time and evidence. Excessive early enthusiasm on the implementation of novelties is, sooner or later, tapered down by evidence and common sense.

A good example of the importance of randomized controlled trials (RCTs) to evaluate innovation is minimally invasive surgery in early cervical cancer. Despite being implemented as an innovative approach in the past 15 years, current RCTs have shown that, actually, open surgery has a better overall survival ${ }^{6}$.
When the effect of interventions is quite moderate, randomization is crucial to demonstrate benefit or, at least, show that we are not doing harm. Non-randomized studies are not the way to solve the difficulties related to RCTs. Admittedly, they might be expensive, difficult to design and bring to term, but currently, RCTs are our best approach to answer questions where the effects of intervention are moderate, and the benefit might not be as evident. The surgeon must stick with the truth and recognize the intrinsic bias that we can have, when we are interested in implementing innovative interventions.

Technology, Apps, Al, can make the life of a surgeon easier to focus on the human experience; rebuild the relationship with patients and their families, giving more time to this important step of care. Setting the expectations before surgery is crucial to understand what the patient wants. As shown recently, our medicine-centered outcomes are not what really matters for a patient; a recent qualitative survey showed that relevant elements for recovery are the return to habits and routines, resolution of symptoms, overcoming mental strains, regaining independence, and enjoying life. This is very different from our doctor-centered outcomes, including overall survival, response, disease-free survival, complications, and length of stay ${ }^{7}$. Perhaps, the future of research in surgery should focus more on what the patient really cares for.

Accessible, affordable, high-quality surgery is also an unmet need in the present that should be addressed. Because of inadequate surgical care, case fatality is high among surgically treatable conditions such as appendicitis, hernias, fractures, breast, and cervical cancer. It is estimated that the lack of investment in surgical care among low-middle-income countries will have an impact of US\$12.3 trillion of economic losses between 2015 and $2030^{8}$. Our health systems should focus on how to improve the investments in health care. Shall we all embrace technology and robotics? Are they a priority? In which hospitals and surgical procedures should they be used? In the same vein, economic and cost-benefit analyses should be a priority on the way we plan ahead to improve surgical care. Information technology is an underestimated resource to aid the physician in improving the care of patients and help them navigate the health system. Apps are used to track vital signs, post-operative complications, and compliance with medical treatment. 
In addition, a simple WhatsApp chat can help to overcome barriers of access and make more efficient the navigation process of patients in a fragmented and disorganized health system. The severe acute respiratory syndrome coronavirus 2 pandemic has imposed a series of changes in the Mexican health system, such as the interhospital reference coordination, that must persist as a quality improvement intervention.

\section{CONCLUSIONS}

The future of surgery lies in the fulfillment of four main conditions: safety, access, efficiency, and efficacy. Innovation and technology should help to achieve them, but must not be the center of our practice. Surgeons should benefit from technological advantages and not forget the current unmet needs of surgical care.

\section{REFERENCES}

1. Gawande A. Two hundred years of surgery. N Engl J Med. 2012;366:1716-23

2. Sheetz KH, Norton EC, Dimick JB, Regenbogen SE. Perioperative outcomes and trends in the use of robotic colectomy for medicare beneficiaries from 2010 through 2016. JAMA Surg. 2020; 155:41-9.

3. Jayne D, Pigazzi A, Marshall H, Croft J, Corrigan N, Copeland J. et al. Effect of robotic-assisted vs conventional laparoscopic surgery on risk of conversion to open laparotomy among patients undergoing resection for rectal cancer: the ROLARR randomized clinical trial. JAMA. 2017:318:1569-80.

4. Hoffman AB, Myneni AA, Towle-Miller LM, Karim SA,Train AT, Burstein M, et al. The early (2009-2017) experience with robotassisted cholecystectomy in New York state. Ann Surg. 2021 [Epub ahead of print].

5. Patel SV, Yu D, Elsolh B, Goldacre BM, Nash GM. Assessment of conflicts of interest in robotic surgical studies: validating author's declarations with the open payments database. Ann Surg. 2018;268:86-92

6. Ramirez PT, Frumovitz M, Pareja R, Lopez A, Vieira M, Ribeiro R, et al. Minimally invasive versus abdominal radical hysterectomy for cervical cancer. N Engl J Med. 2018;379:1895-904.

7. Rajabiyazdi F, Alam R, Pal A, Montanez J, Law S, Pecorelli N, et al. Understanding the meaning of recovery to patients undergoing abdominal surgery. JAMA Surg. 2021 [Epub ahead of print].

8. Meara JG, Greenberg SL. The lancet commission on global surgery global surgery 2030: evidence and solutions for achieving health, welfare and economic development. Surgery. 2015, 157:834-5. 